\title{
Adopciones monoparentales de niños y niñas con «necesidades especiales»: entre el déficit y el empoderamiento
}

Irene Salvo Agoglia

Universidad Alberto Hurtado

isalvo@uahurtado.cl

\section{María Isabel Jociles Rubio}

Universidad Complutense de Madrid jociles@cps.ucm.es
Recepción: 10-10-2017

Aceptación: 21-09-2018

Publicación: 30-05-2019

\section{Resumen}

Las políticas y prácticas adoptivas establecen explícita y tácitamente un matching entre niños y solicitantes con determinadas características. En el caso de las adopciones monoparentales de niños mayores y/o con otras necesidades especiales, ambas partes suelen ubicarse desde un inicio en una posición de menor prioridad. A partir de los resultados de dos investigaciones cualitativas realizadas en Chile y España con adoptantes monoparentales, analizamos sus heterogéneas motivaciones y preferencias respecto de los perfiles de los niños que esperan adoptar y las estrategias que desarrollan frente a los criterios técnicos, discursos e intervenciones profesionales, especialmente en los procesos de evaluación de idoneidad y asignación. Discutimos la necesidad de desarrollar enfoques, criterios e intervenciones centrados en las capacidades y recursos de sujetos que son a priori considerados más vulnerables o estigmatizados en los procesos adoptivos, así como la necesidad y obligación política e institucional en la provisión de apoyos y recursos de acompañamiento en la fase pre y post-adoptiva.

Palabras clave: adopción; monoparentalidad; niños mayores; idoneidad; matching 


\begin{abstract}
Single-parent adoptions of children with "special needs": Between deficit and empowerment
\end{abstract}

Policies and practices of domestic and intercountry adoption explicitly and tacitly establish a matching between older children — or with some type of special need—and single-parent applicants, both of which are usually given lower priority. Based on the results of two qualitative research studies conducted in Chile and Spain with single adoptive mothers, we analyze the heterogeneous motivations and preferences of adopters with respect to the profiles of children declared adoptable, the meanings attributed to technical criteria and professional interventions, especially in the suitability and matching processes. The traditional perspective that considers single-parenthood as a risk factor and this profile of children as the most vulnerable reproduces practices of stigmatization and discrimination. We discuss the need to develop approaches, criteria and interventions focused on the capacities and resources of individuals who have been generally stigmatized in adoptive processes, as well as the political and institutional need and obligation to provide support and support resources in the pre- and post-adoptive phase.

Keywords: adoption; single mother by choice; older children; suitability; matching

\title{
Sumario
}

$\begin{aligned} \text { 1. Introducción } & \text { 4. Resultados } \\ \text { 2. Marco teórico } & \text { 5. Discusión y conclusiones } \\ \text { 3. Metodología } & \text { Referencias bibliográficas }\end{aligned}$

\section{Introducción ${ }^{1}$}

El tradicional escenario en el que jóvenes matrimonios heterosexuales con problemáticas de infertilidad adoptaban bebés recién nacidos se redefinió radicalmente en las últimas décadas. Hoy en día existe un amplio y heterogéneo espectro de configuraciones familiares adoptivas derivadas de las múltiples y complejas transformaciones en los perfiles de niñas y niños ${ }^{2}$ declarados adoptables y de solicitantes ${ }^{3}$, así como de las razones que motivaron la declaración

1. Este artículo se enmarca en dos investigaciones desarrolladas en Chile y España. La primera de ellas titulada «Narrativas de mujeres que han adoptado individualmente en Chile: tensiones, desafíos y perspectivas», financiada gracias al Fondo Interno de la Dirección de Investigación y Publicaciones (DIP), de la Universidad Alberto Hurtado, cuya IP es la primera autora de este artículo. La segunda se tituló «Monoparentalidad por elección: estrategias de autodefinición, distinción y legitimación de nuevos modelos familiares» (FEM200907717) y fue financiada por el Ministerio de Ciencia e Innovación de España, cuya IP es la segunda autora de este artículo. Las autoras agradecen a las instituciones y agrupaciones que colaboraron en los estudios y a las participantes que relataron sus experiencias de vida.

2. Por razones de fluidez en la lectura, utilizaremos el término niños para englobar a niñas y niños dentro de esa categoría.

3. Hablaremos de «las solicitantes» ya que la totalidad de participantes, en uno de los estudios, y la mayoría, en el otro, fueron mujeres. No obstante, algunos resultados pueden resultar aplicables al caso de los varones que adoptan sin pareja, situación que está emergiendo en ambos países. 
de su adoptabilidad. Tras el denominado «boom de la adopción internacional», producido en la primera década del siglo XXI, a partir del año 2005, las cifras de adopción internacional han mostrado una caída paulatina a nivel global (Selman, 2012, 2016). La transformación de estos perfiles implica la necesidad de responder a los desafíos presentes y futuros con aproximaciones interdisciplinarias que consideren la complejidad y diversidad de las necesidades y experiencias de todos los sujetos involucrados en el proceso adoptivo.

En este artículo, presentamos aquellos aspectos comunes de los resultados de dos investigaciones cualitativas que exploraron y analizaron las experiencias de mujeres que adoptaron monoparentalmente en Chile y en España. Los dos países analizados muestran un escenario heterogéneo derivado de un conjunto de transformaciones socioculturales y del impacto de la aplicación de nuevos enfoques, legislaciones y políticas de infancia, tras la ratificación de la Convención Internacional de los Derechos del Niño (1989) y el Convenio de la Haya relativo a la Protección del Niño y a la Cooperación en materia de Adopción Internacional (1993).

Siguiendo las tendencias globales, para el caso de ambos países podría preverse que, durante los próximos años, las adopciones internacionales continúen disminuyendo, las nacionales aumentando y ambas volviéndose cada vez más complejas en razón de los nuevos perfiles de niños y solicitantes. Esta proyección también hace pensar en un incremento de las adopciones monoparentales de niños considerados mayores y/o con «necesidades especiales». De esta forma, pretendemos problematizar algunos desafíos, tensiones y perspectivas en un momento clave para los sistemas adoptivos de ambos países, trascendiendo las especificidades derivadas de la adopción nacional o internacional. Para ello, nos aproximamos a las perspectivas de las adoptantes monoparentales con el propósito de conocer y comprender las experiencias, expectativas, motivaciones y necesidades respecto de los niños adoptables que presentan estos perfiles.

\section{Marco teórico}

\subsection{La construcción de las adopciones monoparentales como factor de riesgo}

En términos más generales, la creciente visibilización de las familias monoparentales ha impulsado su estudio y una incipiente desestigmatización. La evidencia científica disponible muestra que constituyen un grupo heterogéneo en cuanto al nivel de apoyo que tienen, el número de niños que adoptan, las razones de su soltería, las motivaciones para adoptar y las competencias parentales (Pakizegi, 2012). Estas adoptantes viven experiencias de crianza sustancialmente similares a las parejas que adoptan (Feigelman y Silverman, 1977; Shireman y Johnson, 1985), sus problemáticas no difieren de las que pueden presentarse en estas (Tan y Baggerly, 2009), y tienden a tener el mismo éxito en su desempeño parental (Feigelman y Silverman, 1977; Groze, 1991; Pakizegi, 2007). Al mismo tiempo, existe evidencia de que estas adoptantes pueden ser más capaces de manejar la adopción de niños mayores o con algún 
tipo de necesidad especial (Cowan, 2004; Groze y Rosenthal, 1991; Haugaard et. al, 1999; Shireman, 1994), o pueden ofrecer más oportunidades de desarrollo positivo y creatividad (Pakizegi, 2012). Otros estudios refieren que estas solicitantes cuentan con amplias redes de apoyo social en sus comunidades, familias y grupos de amigos - más amplias incluso que las familias biparentales-, y que se sienten altamente satisfechas con la calidad de la respuesta de estos entornos hacia sus necesidades (Díez et al., 2016; Shireman, 1994), así como con su vida, en general, y con su maternidad, en particular (Díez et al., 2016). De esta forma, la investigación concluye claramente que este tipo de adopciones no constituye un factor de riesgo per se para el éxito de las adopciones, ni tampoco para el bienestar de los niños (Cowan, 2004; Feigelman y Silverman, 1977; Kagan y Reid, 1986; Díez et al., 2016; Dougherty, 1978; Shireman, 1994; Shireman y Johnson, 1985; Haugaard et al., 1999; Pakizegi, 2007; Rosser y Moya, 2001). Pese a los hallazgos mencionados, aun parecen persistir creencias ligadas a que la monoparentalidad podría privar al niño de una referencia masculina estable y a que estas mujeres no desean a un hijo en sí mismo, sino como medio para poner fin a su soledad y garantizarse una compañía y un cuidado futuros, las cuales son argumentadas para la denegación de estas solicitudes (Cadoret, 2003; Jociles et al., 2012).

En ambos países donde realizamos trabajo de campo existe un marco legislativo que contempla la adopción por parte de personas sin pareja. Sin embargo, aún observamos el predominio de concepciones deficitarias respecto de la monoparentalidad en la adopción (Jociles et al., 2012; Medina, 2013; Salvo Agoglia, 2018). En el caso chileno, el orden de prelación legal vigente muestra la persistente jerarquización de la estructura biparental sobre la monoparental, que ubica a las personas solteras, divorciadas o viudas en el último lugar del orden de prelación legal, por detrás de los matrimonios nacionales y extranjeros. De esta forma, la adopción monoparental es un fenómeno atravesado por diversas barreras, lo cual se aprecia también en las cifras. Las estadísticas disponibles del período 2017 muestran que, de un total de 901 personas declaradas idóneas vigentes, $5(0,6 \%)$ fueron divorciadas, $64(7,1 \%)$ fueron solteras y 1 era viuda $(0,1 \%)$. Asimismo, del total de los enlaces adoptivos concretados durante el año $2017(n=387)$, las personas con un estado civil no matrimonial aportaron marginalmente al universo de casos registrados, correspondiendo solo a 20 casos $(5,2 \%)$ (SENAME, 2018). Una publicación anterior (Salvo Agoglia, 2018) muestra el trato diferencial y discriminatorio que experimentan algunas de estas adoptantes cuando transitan por el dispositivo técnico y judicial, lo que da cuenta de los imaginarios que recaen comúnmente sobre este tipo de adopción, así como lo perseverantes y luchadoras que deben ser estas solicitantes durante este trayecto.

En el caso español, estas adopciones han tenido un crecimiento progresivo. Por ejemplo, para el período 2000-2004, un estudio realizado por González et al. (2007) estimó que el porcentaje de adopciones monoparentales internacionales se situaba en un 9,4\%. Rodríguez y Jareño (2015) exploraron las características, las opiniones, las actitudes y los comportamientos sobre la 
adopción en una muestra no probabilística de 230 madres y padres españoles que adoptaron internacionalmente, e identificaron que las categorías soltero o soltera y convivencia con pareja [siendo solteros] representaban el 12,2\%, lo que muestra un aumento de estas configuraciones familiares. Un aspecto interesante es que el porcentaje de cohabitación de estos solteros resultó ser muy bajo (1,3\%). De hecho, el 14,3\% de las madres y padres adoptivos decidieron contraer matrimonio precisamente para cumplir con este requisito exigido en algunos países de origen de los niños, alcanzándose así un 15,6\% de cohabitación, lo que evidencia la jerarquización de la conyugalidad y de la biparentalidad, y que casarse constituye una estrategia de ajuste a las reglas del mercado de la adopción internacional (Rodríguez y Jareño, 2015). Otros estudios españoles que analizan la variable de la estructura familiar a la hora de comprender la ruptura de las adopciones ${ }^{4}$ pueden tornarse algo controvertidos al sugerir explícita o implícitamente que la monoparentalidad podría constituir un factor de riesgo para ese truncamiento (Berástegui, 2003; García y Grau, 2016). Habría que analizar si estos estudios contienen ciertos sesgos metodológicos y/o de interpretación que lleven a extraer dichas conclusiones, y vigilar que no se sobreinterpretara la relación existente entre estructura familiar y la dicotomía éxito/fracaso de la adopción, ni se retroalimentara — entre profesionales e investigadores - una concepción carencial de estas adopciones que pudiera estar motivada por dichos sesgos y/o por motivos ideológicos (Jociles et al., 2012).

\subsection{La construcción de las «adopciones especiales» como factor de riesgo}

En cuanto a los perfiles de niños susceptibles de ser adoptados, encontramos algunas tendencias comunes en ambos países. En Chile, el desglose estadístico de las adopciones nacionales e internacionales por grupos de edad muestra la tendencia sostenida al aumento de la edad al momento de la colocación adoptiva. En el caso de las adopciones nacionales, el grupo conformado por niños entre 0-1 año representó el 32,8\%; el de 1-3 años, el 30,3\%; el de 4-7 años, el 24,8\%, y el de mayores de 8 años, el 5,1\% (SENAME, 2018). En cuanto a las adopciones internacionales, se ha establecido como perfil único de niños postulados a adopciones internacionales el de aquellos que son mayores de 4 años de edad, lo que se encuentra asociado a la problemática de la institucionalización de la infancia en este país. De esta forma, los niños entre 1 y 3 años representan el 1\%; los de 4-7, el 58,8\%, y los mayores de 8 años, el $40,2 \%$ (SENAME, 2018). Pese a los esfuerzos desplegados por los equipos profesionales durante los últimos años, resulta muy difícil encontrar familias interesadas

4. El concepto de adopción truncada o rota es complejo y controvertido. No obstante, suele entenderse en España como «aquella adopción plena en la que, como resultado de una situación de desamparo, la administración pública asume la tutela del menor sobreviniendo un cese de la convivencia entre los progenitores [sic] y su/s hijo/s adoptivo/s» (García y Grau, 2016: 33). 
en adoptar, en Chile o en el extranjero, a centenares de niños calificados de «perfil prioritario» (Galleguillos, 2015). En España, las cifras de adopciones nacionales e internacionales por grupos de edad evidencian que los niños en condiciones de adoptabilidad son también de mayor edad. De este modo, el grupo conformado por niños mayores de 4 años representó en el año 2015 el 37\% de las adopciones nacionales, porcentaje que aumentó al 45,7\% al considerar la adopción internacional (MSSSI, 2017b). Sin embargo, el porcentaje de estas adopciones se sitúa muy por debajo de los Países Bajos (Rodríguez y Jareño, 2015), en los que muchos solicitantes se ofrecen para adoptar niños mayores, con alguna discapacidad o con enfermedades crónicas.

Tradicionalmente, el término «adopciones especiales» englobó la adopción de grupos de hermanos, de niños considerados «mayores» o con problemáticas de salud o de comportamiento, o bien, pertenecientes a determinados grupos étnicos, asumiéndose que las adopciones comunes o «normales» eran de bebés recién nacidos o de corta edad y considerados sanos. Dados los cambios operados en la demografía de la adopción, esta clásica distinción ha ido perdiendo sentido y utilidad, y el propio concepto resulta confuso y ambiguo (Palacios, 2007). El uso aún generalizado de esta noción encierra en su propia definición ciertos sesgos que resultan cada vez menos aplicables. Además, sitúa a los niños en un rol pasivo, abonando la construcción de una imagen de vulnerabilidad y falta de agencia (Jociles et. al., 2012). Para el caso específico de las adopciones de niños con algún tipo de discapacidad, estas son atravesadas por discursos de déficit e impedimento que proporcionan representaciones engañosas de las condiciones reales de estos niños, lo que afecta sus posibilidades de adopción (Bunt, 2013; Cousins, 2009).

La producción científica sobre las tradicionalmente denominadas «adopciones especiales» y, específicamente, sobre las consideradas «tardías» o de niños en edades mayores tiene una larga tradición en países anglosajones (Barth, 1991; Forbes y Dziegielewski, 2003; McRoy, 1999; O’Dell et al., 2015; Julian, 2013; Leung et al., 2005). Existe bastante unanimidad respecto del rol crítico que desempeña la edad del niño al momento de su colocación, siendo muy disímil la definición de cuándo se es un «niño mayor». Algunos estudios exploran longitudinalmente el impacto de la edad del niño al momento de la colocación en la tasa de interrupción de las adopciones, pareciendo existir una considerable correlación entre niños más pequeños y menores posibilidades de interrupción o disolución y, por lo tanto, encontrándose mayor riesgo en las adopciones de niños mayores (Coakley y Berrick, 2008; Julian, 2013; Paniagua et al., 2016; Rosenthal et al., 1988). Por supuesto, las rupturas no son precipitadas por la presencia aislada de un solo factor de riesgo, sino por la influencia conjunta y compleja de múltiples factores de riesgo acumulados a través del tiempo en una misma situación familiar (Paniagua et. al. 2016). La investigación sobre el tópico aún es escasa en el contexto iberoamericano y resulta importante seguir fortaleciendo la evidencia al respecto (Berástegui, 2012; Amaral y RossettiFerreira, 2009; Ebrahim, 2001; Otuka et al., 2013, Paniagua et al., 2016; Silva y Benetti, 2015). 
Pese al progresivo aumento de la edad de los niños al momento de ser declarados adoptables, estos perfiles no se ajustan a las expectativas o preferencias de la gran mayoría de los solicitantes, quienes todavía buscan incorporar a sus familias un hijo lo más pequeño y saludable posible (Berástegui, 2012; Brind, 2008; Bunt, 2013; Rodríguez y Jareño, 2015). Los solicitantes esgrimen diversas razones: que su experiencia de parentalidad sea lo más parecida posible a la de la parentalidad biológica, que los niños mayores tendrán más problemas que los más pequeños y/o que desean influir más directamente en el desarrollo de su personalidad (Brind, 2008). Bajo estos argumentos subyace un paradigma biogenético del parentesco y un conjunto de ideas negativas o estereotipadas acerca de los niños adoptables que son diferentes a los inicialmente esperados (Brind, 2008; Bunt, 2013). No obstante, la investigación ha mostrado que un adecuado proceso de clarificación de expectativas y formación, así como la disposición de recursos de apoyo post-adoptivos, es clave para el éxito y el incremento del nivel de satisfacción en estas adopciones (Forbes y Dziegielewski, 2003; O’Dell et. al. 2015).

\subsection{La asignación de niños mayores a adoptantes monoparentales: ¿interés superior del niño o supremacía de un modelo familiar hegemónico?}

$\mathrm{Al}$ igual que en otros países, en Chile y España, el Estado y los organismos que intermedian las adopciones y los profesionales especializados informan desde un inicio a los solicitantes sobre los cambios de perfil de los niños producidos en las últimas décadas, así como los desafíos derivados de ello, planteando como deseable la flexibilización de las expectativas en torno a la edad u otras características del niño. Junto con ello, los profesionales también tienen el desafío de evaluar a solicitantes que no están motivados ni convencidos de adoptar a niños con los perfiles antes descritos.

La asignación o matching entre una familia y un niño determinado constituye un proceso de toma de decisiones técnicas altamente subjetivo (Hanna y McRoy, 2011), lo cual se ve incrementado por la valoración diferencial de las configuraciones familiares de las solicitantes. La investigación sobre familias que adoptan a niños con «necesidades especiales» se ha centrado principalmente en parejas heterosexuales (Leung et al., 2005), por ser las mayoritarias. No obstante, diversos estudios muestran que, por lo general, a las adoptantes monoparentales se las propone para la adopción de niños de más edad más frecuentemente que a los solicitantes matrimoniales (Aoki, 1977; Groze y Rosenthal, 1991; Feigelman y Silverman, 1977; Shireman, 1994; Pena, 2016), constituyendo una categoría de destinatarios «por defecto" de determinados perfiles de niños menos solicitados (Poveda et al., 2013).

Los prejuicios ideológicos sobre la monoparentalidad, que establecen una mayor jerarquía de la estructura biparental al momento del matching, se visibilizan también cuando, enfrentados a la determinación de la adoptabilidad de un niño, se recurre tanto a razones vinculadas con la «desestructuración» de su familia de origen (por ser monoparental) como a una supuesta preferencia 
universal según la cual todo niño desearía formar parte de una familia biparental. Por lo general, se trata de una cuestión dictaminada por adultos, sin considerarse la opinión de los niños involucrados, quienes tienen escasa o nula posibilidad de elegir el tipo de familia a la que quieren integrarse (Poveda et. al., 2013). La presunción de que todo niño preferiría una familia constituida por un padre y una madre, y que garantizar su "Interés Superior» implica asignarlo necesaria o preferentemente a una familia biparental, deja entrever un sesgo ideológico fundamentado implícitamente en lo que Memmi (2014) ha llamado una "naturalización de la psique humana», es decir, un constructo universal e independiente de cualquier circunstancia o consideración de tiempo, lugar o experiencia social y personal.

Junto a lo anterior, la existencia del llamado adoption marketplace (Blackstone et. al, 2008; Högbacka, 2008; Raleigh, 2012, Poveda et al., 2013) hace comprensible que aquellos niños menos demandados tengan más probabilidades de ser asignados a estructuras familiares menos valoradas. La elegibilidad de la familia más apropiada para estos niños vendría determinada por criterios de oferta y demanda, de manera que, si no existen suficientes familias "con padre y madre» dispuestas a aceptarlos, se asignarían a solicitantes monoparentales, quienes ocupan una posición marginal en este mercado (Jociles et. al, 2012). Las fuerzas de este mercado también impactarían los aspectos más íntimos de las decisiones de estas solicitantes, quienes se ofrecen más frecuentemente para adoptar a niños con los perfiles mencionados, ajustando sus esperanzas subjetivas a las probabilidades objetivas que tienen en ese mercado (Bourdieu, 1991: 94). Este ajuste es, a menudo, mediado y reforzado por profesionales que pueden presionarlas a reconsiderar sus expectativas iniciales respecto a la edad máxima u otras características de los niños que desean adoptar. Según Brind (2008), algunos trabajadores sociales consideran que, en ocasiones, las adoptantes monoparentales aceptan adoptar un niño más grande por razones prácticas, pero probablemente querrían un hijo más pequeño.

\section{4. "Motivación por la solidaridad» en adopciones de niños mayores: ambivalencias y deslindamientos}

Otro foco de interés en las investigaciones sobre las adopciones monoparentales ha sido el de la denominada «motivación por la solidaridad», aducida por las solicitantes individuales para explicar por qué se decantaron por la adopción. Jociles (2013: 214) plantea que estas mujeres «no adoptan —al menos no exclusivamente- por no tener pareja, por encontrarse solas o por no tener otras vías para acceder a la maternidad», sino que porque esta vía de acceso a la maternidad conlleva la satisfacción de las necesidades de un niño cuya familia de origen no lo pudo cuidar, cuestión que da sentido a su elección y contrarresta la representación carencial que subsiste en el imaginario social con respecto a ellas. Una imagen que, por otro lado, contrasta con su autorepresentación como mujeres «autónomas, fuertes, luchadoras, independientes y que de este modo tienen mucho que dar» (Jociles, 2013: 214). No 
obstante, esta motivación es cuestionada y considerada disfuncional por el discurso experto para todo tipo de solicitantes, llegando incluso a constituir una posible causa de denegación de la idoneidad (Jociles y Charro, 2008; Jociles, 2013; Marre, 2009).

Según Ebrahim (2001), en el caso de las solicitantes monoparentales, habitualmente consideradas «tardías» (por ser mayores de 40 años de edad), existiría una marcada presencia de argumentos de corte solidario y altruistas en sus motivaciones para adoptar, más que en los casos de quienes desean adoptar bebés. Estas solicitantes expresarían una elevada sensibilización respecto de la situación de abandono o institucionalización de estos niños, ligada en ocasiones a valores religiosos (Ebrahim, 2001), siendo las mujeres más religiosas las que más aceptarían este tipo de adopción (Van Laningham et al., 2012). Para Cowan (2004), tanto la monoparentalidad como la práctica religiosa por parte de las madres adoptivas - independientemente de la estructura familiarserían dos de los factores que promoverían un contexto de mayor estabilidad, de manera que la adopción de estos niños sería una forma de extensión de sus sentimientos religiosos, lo que consecuentemente otorgaría una mayor probabilidad de éxito de las adopciones de niños mayores, especialmente cuando presentan "problemas emocionales o discapacidades físicas» (p. 1016). Esta autora considera un factor protector la participación de estas madres en una Iglesia, entre otras razones, por la red social que logran construir a raíz de esta participación (Cowan, 2004). Si bien los datos aportados por Cowan (2004) resultan insuficientes y se precisaría de una investigación de mayor profundidad para apoyar esta hipótesis, hay indicios que la confirman o, al menos, no la refutan. El análisis realizado por Berástegui (2003: 118) sobre la relación entre motivaciones para adoptar y tasas de ruptura muestra que las motivaciones «humanitarias, filantrópicas o religiosas» no se corresponden con las tasas de ruptura más altas, sino que ocupan un modesto tercer lugar entre los cuatro tipos de motivaciones consideradas por la autora. Justamente, son motivaciones centradas en las necesidades de protección de los niños las que se consideran más beneficiosas para el curso del proceso adoptivo (Coakley y Berrick, 2008). Todo lo anterior no solo pone entre paréntesis que este tipo de motivaciones sea considerada disfuncional por los técnicos y represente algún riesgo para el éxito de las adopciones, sino que permite pensar que en algunos casos -con los recursos y apoyos necesarios- puede incluso contribuir a adopciones con muy buenos resultados.

\section{Metodología}

Ambos estudios se basaron en un enfoque cualitativo (Vasilachis de Gialdino, 2009) y etnográfico (Agar, 2006). Las características mayoritarias de las participantes en ambos estudios fueron coincidentes con las identificadas en otras investigaciones sobre el mismo colectivo (Bock, 2000; Ben-Ari y Weinberg-Kurnik, 2007), es decir, ser madres a edades mayores, tener un elevado nivel académico, ser económicamente solventes o ubicarse entre las 
clases medias-altas. En la investigación desarrollada en Chile (2013-2014), el grupo se conformó por 10 mujeres heterosexuales que habían adoptado nacionalmente como mínimo hacía dos años, durante el período comprendido entre 1999-2012. En el momento de la entrevista, se encontraban en un rango etario promedio de 46,5 años y residían en las ciudades de Santiago y Viña del Mar; 8 eran solteras y 2 divorciadas, de las cuales una convivía con su pareja heterosexual y su hija. Todas manifestaron ser económicamente autónomas, y 9 contaban con estudios universitarios. Respecto de las edades de adopción de sus hijos, en 6 casos predominó la adopción de niños mayores; no obstante, en 4 casos adoptaron a sus hijos de bebés (de 3 días; 1 mes; 6 meses; 10 meses de edad).

En el estudio llevado a cabo en España (2008-2010), con trabajo de campo en Madrid, Cataluña y la Comunidad Valenciana, se incluyó a mujeres que se encontraban en distintos momentos de su proyecto de adopción (en proceso de postulación / habiendo ya adoptado) y que hubieran elegido diversos países para adoptar. De esta manera, fueron entrevistadas 58 adoptantes monoparentales, de las cuales 56 lo hicieron vía adopción internacional (China, Rusia, Nepal, Etiopía, Vietnam, Perú, México, Haití, entre otros países). Las entrevistadas tenían una media de edad de 40,7 años. En cuanto al nivel académico, el $81,0 \%$ tenía estudios superiores y las demás habían culminado los secundarios. A 15 de ellas les asignaron niños mayores de 3 años (25,9\%), 3 de los cuales presentaban problemas de salud adicionales; a $34(58,1 \%)$ les asignaron niños menores de esa edad, pero 12 de ellos presentaban necesidades especiales considerables; y $9(15,5 \%)$ no tenían aún asignación.

En Chile, el acceso a las entrevistadas se realizó vía institucional (Fundación Chilena de la Adopción y Dirección Nacional de Adopciones de SENAME), lo cual fue complementado con una estrategia de bola de nieve. Luego se estableció contacto con las interesadas mediante correo electrónico o telefónicamente, enviándoles un resumen adaptado del proyecto de investigación y del consentimiento ético informado. En el caso de España, se las invitó a colaborar en las entrevistas mediante llamadas en comunidades virtuales en donde participaban adoptantes monoparentales, así como a través de contactos establecidos en kedadas $^{5}$, reuniones de familias adoptantes, cursos de formación, actividades de post-adopción, Entidades Colaboradoras de Adopción Internacional (ECAI) y Asociaciones de Familias Adoptivas.

En cuanto a las herramientas de producción de información, en ambos estudios se utilizó como instrumento la entrevista cualitativa, y se prepararon guiones organizados según la lógica propia de las entrevistas etnográficas (España) y de las entrevistas narrativas (Chile), estableciendo bloques temáticos donde se incluyeron durante el transcurso de las conversaciones las preguntas adecuadas al desarrollo de las narrativas de las participantes, por ejemplo, sus representaciones sobre los niños adoptables (características, expectativas, desafíos, etcétera). Para analizar el material de campo producido, en el caso

5. Reuniones presenciales de algunas de las comunidades virtuales. 
chileno, se utilizó un método narrativo (Riessman, 2008) y, en el caso de España, se recurrió a la Grounded Theory (Strauss y Corbin, 1998), construyendo categorías analíticas a partir de los discursos y las prácticas de las participantes, trianguladas en un proceso de interanálisis. Los extractos seleccionados fueron identificados mediante un código que resguarda la confidencialidad, a la vez que diferencia las entrevistas realizadas en Chile $(\mathrm{ECH})$ de las realizadas en España (EE).

\section{Resultados}

Los hallazgos que a continuación presentamos no pretenden ser generalizables ni concluyentes, sino dar cuenta de algunas tendencias identificadas en ambos países que nos permiten plantear reflexiones, identificar desafíos y proyectar nuevas perspectivas sobre la temática analizada.

\subsection{Conociendo las "reglas del juego»}

Los resultados muestran que algunos profesionales plantearon desde un inicio a las solicitantes monoparentales que los niños adoptables serían exclusivamente quienes se ajustaran a los perfiles menos solicitados por los adoptantes matrimoniales, o bien que no flexibilizar sus expectativas a este respecto podría extender sus tiempos de espera. En ambos países la disposición inicial de las solicitantes a aceptar niños mayores o "con necesidades especiales» es altamente valorada por los profesionales en el proceso de evaluación de la idoneidad, y por lo tanto, este sería un camino para aumentar sus probabilidades de adoptar. Algunas participantes chilenas y españolas nos relatan que, más temprano que tarde, algunos profesionales les plantean las opciones que están disponibles para ellas en lo que respecta al perfil de los niños adoptables. Junto con ello, muchas veces se les presentan estas asignaciones únicamente desde un prisma negativo o como una consecuencia del menor valor de la oferta para adoptar cuando proviene de ellas o del supuesto deseo universal - ya mencionado - de todo niño de integrarse en una familia biparental, considerada la ideal.

Tú tienes que entender — ni siquiera me preguntó el nombre- que no tienes ninguna posibilidad de adoptar a un niño sano. A una soltera le podemos entregar solamente niños que tengan un síndrome alcohólico fetal. Hace como dos meses, una mujer soltera adoptó a dos niñitas, pero tenían como 15 años. Eso es todo lo que te podemos ofrecer acá, porque a los niños más pequeños preferimos mandarlos a adopción internacional que entregárselos a una soltera. ¿Así que eso es lo que tú piensas? — le dije yo—. Esa es la ley y eso está aquí escrito. ¡Perdón! —le dije- Eso también es una interpretación de la ley. No, esa es la ley —me dijo- _ ¡Ya! —le dije-. Parece que contigo no tengo nada más que hablar. No —me dijo—. Y me fui $(\mathrm{ECH} 9)$.

Y me dijo: ¡Con la cantidad de parejas que quieren niños, se creerá usted que se los van a dar! Y yo dije: Bueno, pero las parejas quieren bebés. Yo no quiero 
bebés, es lo primero que le estoy diciendo, que no quiero bebés porque yo soy consciente de que yo no me puedo dedicar a cuidar bebés. Quiero niños que yo coja de la manita y los lleve al colegio, y que mientras yo trabajo ellos estén en el colegio, y luego yo ya los cuidaré. Total, que la señora me dijo que bueno, que si quería rellenar un chisme (un formulario de solicitud), pero que pensara que no me lo iban a dar nunca (EE22).

Las adoptantes de ambos países subrayan el componente discriminatorio de esta asignación, identificando la contradicción con aquellos discursos jurídicos y técnicos que pretenden asegurar el "Interés Superior del Niño». Esto porque, en tanto que madres más sobrecargadas con dobles jornadas y tareas que no siempre o difícilmente pueden delegar en otros, se les exige hacerse cargo de niños que experimentarían problemáticas de diferente orden de complejidad, que necesitarían mayor atención, dedicación y/o dotación de recursos económicos.

Es muy loco darle precisamente a una persona que está sola una criatura que es más grande y que tiene mucha más necesidad de atención que cualquiera. O sea, ¿cómo una se las va a arreglar para atender en lo concreto y en términos de cumplir todos los roles?, ¿cómo voy a ser jefa de hogar, trabajar con un hijo para mantener la familia y además tener que estar con la presencia y energía psicológica, afectiva y de todo para dedicarle a una criatura que me necesita de manera mucho más especial? Esta ha sido una de las pocas veces en la vida que no me sentí capaz (ECH8).

O bien, se argumenta que son los países solicitantes —en el caso español, con predominio de adopciones internacionales - quienes establecen dichos requisitos.

Yo les dije: Yo creo que sería más normal que a un matrimonio le dieran niños mayores y con necesidades especiales que a una monoparental. Se me quedaban mirando y respondí: Por la simple razón de que vosotros tenéis mucho más tiempo, ya que estos niños necesitan mucha dedicación, y más oportunidades económicas que una persona que está sola. Yo se lo comenté a la psicóloga y ella me dijo: Tienes toda la razón del mundo. Pero los países lo piensan de otra manera (EE52).

De esta manera, tanto en Chile como en España, identificamos en algunos relatos la influencia de imaginarios deficitarios y de riesgo que afectan a estas solicitantes, en la aplicación de criterios técnicos que toman como referente normativo a la familia nuclear, biparental y heterosexual (Jociles y Charro, 2008; Jociles et. al., 2012; Rodríguez y Jareño, 2015; Salvo Agoglia, 2018). Esto conlleva el riesgo de invisibilizar desde un primer momento las fortalezas y capacidades de estas solicitantes para el cuidado y atención de este perfil de niños y, al mismo tiempo, desconocer la agencia que los niños tienen para crear activamente sus familias y sobreponerse a condiciones y experiencias difíciles de sus historias de vida. 


\subsection{Negociando expectativas y motivaciones en relación al perfil de los niños adoptables}

Ante las reglas del juego planteadas inicialmente, algunas solicitantes manifiestan las expectativas y razones que tienen para adoptar un hijo lo más pequeño posible, las aprehensiones ante las posibles dificultades de vinculación o de cuidado que podría tener un niño más mayor o que presente necesidades añadidas, o bien expresan no querer enfrentar desafíos adicionales que pudieran determinar una experiencia de maternidad sobrecargada de exigencias, con pocas gratificaciones y espacios de disfrute. Además, aducen que estos niños han vivido situaciones eventualmente traumáticas, como maltrato, abandono e institucionalización, que podrían haber dejado en ellos una huella imborrable y difícilmente reversible.

Está el otro lado más importante, que es el lado de ellos, que no hay derecho que vivan sin familia hasta tan grandes y pasen por todo lo que pasa un niño antes de ser adoptado, porque evidentemente queda cierta huella que deja el hecho de haber tenido un tiempo de la vida sin un vínculo exclusivo, sin una pauta de apego temprana; $y$, si eso se prolonga en el tiempo, es terriblemente dañino (ECH6).

La obsesión de que iba a ser muy mayor, aunque me gustaría ahorrarme los pañales, ¿sabes? (risa), pero era como decir: ¡Jolín! Toda la vida esperando para ser madre, ¿y ahora me viene criado? (...) Yo no sé el abandono a los 2 años cómo marca. ¿Y si tiene 3 y pico y recuerda a sus padres? No sé la sensación o la inseguridad que te transmite el que te hayan dejado allí abandonado, ¿no? Ahí hay como una cuerda floja. Aunque luego venga alguien a darte mucho cariño, ¿hasta qué punto tú te aferras a crear lazos con esa persona? Yo no sé si funcionaría, si el niño encontrará en mí eso, ni qué secuelas deja eso (EE18). ${ }^{6}$

Otras entrevistadas expresan su deseo de querer y poder vivir la infancia de sus hijos durante un mayor número de años.

Creo absolutamente que sería deseable siempre que sean más pequeños, desde el punto que uno lo mire. Desde la dimensión más egoísta, personal «pucha que rico", es distinto y es importante saltarse las menos etapas posibles de la vida de un hijo (ECH6).

Está claro que te llama estar el mayor número de años posible con un niño pequeño, porque los años de la infancia son muy ricos. Pero, bueno, mi cabeza, según lo que haya decidido (decidió ampliar su certificado de idoneidad - CI - hasta los 5 años), saca ventajas de lo que haya decidido; entonces, te pierdes dos o tres años que son una maravilla, pero ¡bueno! (EE9).

6. En las viñetas se aprecia una apropiación de discursos expertos y sociales sobre «el abandono» y las consecuencias de "la institucionalización», que estas mujeres utilizan para resistirse a las asignaciones que les ofrecen. 
Las posiciones de las adoptantes frente a los perfiles de niños que les podrían ser propuestos para asignación son heterogéneas. En algunos relatos identificamos cómo opera la lógica del adoption marketplace (Raleigh, 2012), que incrementa su disposición a aceptar niños con estos perfiles suponiendo que, si abren esta alternativa, aumentarán sus probabilidades de ser elegidas y disminuirán sus tiempos de espera.

El CI lo podría haber hecho hasta 3 años, de 1 a 3 años, y yo decidí abrirlo hasta 5. Y yo pienso que, si lo abres hasta 5, te darán [un niño] de 5. Bueno, ¡ya se verá! Generalmente los que tienen más dificultades para ser adoptados son los más mayores, entonces, supongo que hay más facilidad para que nos los asignen o para que [el periodo de espera] pueda ser más corto (EE9).

Aun cuando la mayor flexibilidad respecto del perfil de niños adoptables puede ser una forma de entrar en «las reglas del juego», esto no impide que algunas solicitantes reconozcan ciertas ventajas en la adopción de niños más mayores, relacionadas con la menor distancia entre su edad como madres y la edad de sus hijos. Teniendo en cuenta el retraso de su maternidad, esgrimen motivos pragmáticos y emocionales sobre el tiempo y «la energía» requeridos para el cuidado y la crianza, que deben ser conciliados con su rol de jefas de hogar. Por otro lado, argumentan que los mayores grados de autonomía que presentan los niños más grandes pueden resultar favorables para trabajar y organizar la logística doméstica mientras estos asisten al nivel preescolar o a la escuela.

Si tengo, por ejemplo, 55 años y digo: Quiero un bebé; pues igual me dicen: No, tiene que ser de 4 a 7 años. Y, en mi caso, yo quería un niño mayor. Por mi edad, porque cuando empecé tenía 39 años, podría haber sido perfectamente un bebé, pero no quería un bebé, y entonces a mí me pusieron lo que yo quería. Pedí de 3 a 6 porque ya son un poquito más independientes, ya se puede jugar más. Además, por mis circunstancias, un bebé... no puedo. Un poco por todo: tema laboral, económico... (Si es) un bebé, tienes que tener a otra persona en casa o hay que estar con guarderías. Y un niño de 3 años o de 6 ya puede ir al cole, y eso sí que dentro de mi vida lo puedo llevar mejor que un bebé (EE15).

A veces, son convicciones que expresan desde el inicio del proceso, o bien responden a una aceptación o adecuación al contexto evaluativo y la propuesta técnica de asignación, ante la que resignifican como aspectos positivos las eventuales desventajas de adoptar un niño con este perfil. En estos casos, sus valoraciones se dirimen según la lógica de que cada experiencia busca su propia congruencia en lo vivido.

Cada una, en la situación y circunstancia que le toca, trata de centrarse en el aspecto mejor de esa experiencia y sacarle el aspecto positivo (ECH7).

En otros casos, parece ser cuestión de hacer de la necesidad una virtud, viendo ventajas donde antes no se veían. 
Por mi edad, a mí me pusieron de 1 a 3 años, y cuando me asignaron la niña, mi niña tenía 3 años y medio, entonces, al principio pensaba: Es que ya es muy mayor, es que no sé qué, es que no sé cuántos, como que había roto mis esquemas, ¿no?, de que yo la quería más pequeña, pero luego la verdad es que pensándolo digo: ¡Ah, pues, si son todo ventajas!, ¡quita, quita! $\mathrm{Y}$, entonces, ya estaba tan contenta con que la niña tuviera 3 años y medio (EE24).

Otras participantes establecen claramente sus límites, sobre todo cuando enfrentan la propuesta de asignación de niños con problemáticas graves de salud que, en el caso chileno, se proponen principalmente a matrimonios extranjeros o adoptantes solteras nacionales y, en el caso español, sobre todo a las solteras o a las parejas del mismo sexo que desean adoptar, nuevamente en coherencia con la lógica del adoption marketplace (Blackstone et. al, 2008; Högbacka, 2008; Raleigh, 2012).

Creo que coherentemente con la ideología de que las solteras no tienen que adoptar, el discurso de esta asistente social era: Las solteras, si adoptan, tienen que adoptar a aquellos niños que fueron rechazados en todas las otras instancias, y niños con problemas severos, niños que son un problema para el Estado. Son palabras de ella, porque ella usó estas palabras (ECH5).

Tú, como monoparental, olvídate de un niño sano, si es que algún día te lo asignan. Ahora, si tú quieres un grupo de hermanos o niños enfermos, ahora mismo tengo unos cuantos expedientes que no los quiere nadie (...) ¿Pero tú te estás dando cuenta de que un niño enfermo depende de mí más de lo que depende un niño sano, y tú me estás dando prioridad (para adoptarlo) sobre una familia de dos, sobre una pareja? ¿No te parece eso un poco contradictorio? ¡Ah!, es lo que hay. No fui la única a la que le dijeron eso. Luego lo puse en el foro iporque salí con un rebote de allí! Y dije que no (EE55).

Otras entrevistadas relatan que el largo tiempo de espera se relacionó con su negativa a adoptar niños con problemáticas graves de salud, lo que actuó como presión aun cuando la gran mayoría de ellas dejó en claro durante su postulación y evaluación de idoneidad que no estaría en condiciones de cuidarlos.

Me llamaron un día para decirme que tenían una guagua (bebé) para mí y me preguntaron: ¿Tú qué entiendes por buena salud? Yo dije: Bueno, para mí buena salud es un niño autónomo, que no está en cama y no tiene ningún requerimiento especial. Eso para mí es un niño con buena salud. Puede ser miope, tener las piernas chuecas, hiperquinético, tener déficit atencional. Esas cosas también son buena salud, no tienen que ver con mala salud. Entonces dijeron: ¡Ya!, es que nosotros tenemos para ofrecerte un niño que no tiene grandes problemas de salud. Es un niñito de 1 año y medio y está internado en un hospital, es oxígeno-dependiente de por vida, tiene retraso psicomotor, tiene sífilis congénita y requiere de tratamiento quinesiológico todos los días para poder respirar, pero tiene súper buena salud. Me quedé callada y confundida, me sentí maltratada, pero igual consulté con una pariente médica, que 
me dijo: Por ningún motivo. Parte de la base que requiere kinesióloga y tubos de oxígeno todos los días, casi todo el día. No te puedes hacer cargo porque tu sueldo de profesora no te alcanza. Luego hablé con la profesional y le dije que no podía adoptar a ese niño. Me hizo sentir muy mal, como si lo estuviera abandonando y no me quisiera hacer cargo, me sentí pésimo. Me estaban ofreciendo una posibilidad y la estaba dejando ir con todo lo que había esperado y anhelado a un hijo. (...) Me dijo que no podía ser que yo estuviera rechazando la oportunidad que me estaban dando para ser mamá y que no podía esperar oportunidades diferentes a esa $(\mathrm{ECH} 5)$.

Observamos que, pese al juicio negativo de algunos profesionales frente al descarte de estas propuestas de asignación, las solicitantes se muestran capaces de esgrimir razones de índole económica, pragmática y de sobrecarga emocional tras un proceso reflexivo y de análisis de sus posibilidades y límites para cuidar adecuadamente a esos niños, junto con la necesidad de preservar que su experiencia de maternidad también sea un espacio gratificante y no solo de sacrificio, esfuerzo y sobreexigencia. Por otro lado, en el análisis retrospectivo que las participantes hacen sobre la adopción de sus hijos con más edad o con problemáticas que consideraron asumibles, plantean que su experiencia ha sido positiva, porque adoptar un hijo más grande les brindó la posibilidad de comunicarse mejor con él y construir una relación positiva entre sí.

El hecho de haber optado por un niño mayor creo que fue más beneficioso para mí porque existía la comunicación y la complicidad. Me cuenta lo que le pasa en el día, yo me quedo más tranquila $(\mathrm{ECH} 4)$.

Ahora, con la renovación he pedido hasta 4 años, que me aumentaran un año más por si acaso, pensado en que, si no sale en Marruecos, pues en Nepal tengo más posibilidad hacia más mayor. Mi sobrina tiene 3 y medio y me encanta ahora la edad que tiene, está genial por lo que razona, porque habla por los codos, las historias que se cuenta y porque puedes ir con ella a todas partes sin depender de pañales, cochecitos ni de chorradas (EE55).

Una adoptante española considera ventajosa la adopción de un niño más grande porque le permitió conocer los orígenes de su hijo gracias a sus recuerdos, estimando que a él se le hizo también «más fácil aceptar su adopción».

Luego, sí es diferente que el niño entre en tu vida a los 4 años, a los 3 o a los 7, a que sea un bebé. (...) Por ejemplo, en este tipo de críos, que tienen memoria, que recuerdan y tal, suele ser más fácil la aceptación de la adopción que en un niño que empieza a elucubrar más adelante: ¿Qué vida he tenido, qué historia he tenido, qué tal? Eso sí que es un error lamentable, que un niño no sepa desde el principio que es adoptado. Ese problema no lo tenemos [las adoptantes de niños mayores] porque lo saben, lo saben ellos. En este caso, yo intento recordarle, que no olvide lo que sabe. Recordarle y que lo viva como algo natural, que no sea para él un problema (EE16). 


\subsection{Replanteando la «motivación de la solidaridad» en relación a los nuevos perfiles de niños adoptables}

Como se ha adelantado, ante las características de los niños propuestos en la asignación, las entrevistadas también elaboran argumentos y posiciones favorables y/o contrarias al respecto. Otro elemento que emerge en las entrevistas realizadas tanto en Chile como en España, es la consciencia de que existen muchos niños privados de cuidados parentales e institucionalizados que necesitan vivir en una familia, lo que contribuye a decidir adoptar a niños con más edad de lo inicialmente esperado.

Afuera hay niños que ya nacieron y están esperando que alguien los acoja, ¿̇te fijas? Tú tienes que ser abierto, y hay que enseñarle a la gente eso, a amar al prójimo, ¿y por qué no a un niño que lo necesita? Él lo único que quiere es eso: protección, amor, cariño $(\mathrm{ECH} 2)$.

Puestos a pensar un poco, habiendo niños que luego realmente cuesta mucho adoptar, entonces, habiendo tantos niños en el mundo que no tienen dónde vivir ni qué comer ni tienen a nadie que los quiera, andar haciendo ese tipo de chanchullos [tener un hijo mediante reproducción asistida con donante de semen], por decirlo de alguna manera, pues tampoco me parece bien (EE17).

Sin embargo, las solicitantes tienen muy claro que resulta altamente problemático argumentar toda razón que pudiera ser entendida por los profesionales como una motivación por la solidaridad.

Daba la sensación [en la formación pre-adoptiva y evaluación de la idoneidad] de que hicieras lo que hicieras estaba mal hecho, ¿no? Cuando una de las preguntas es: ¿Por qué quiere usted adoptar?, y una de las respuestas era: Porque quiero sacar a un niño de la calle o algo así, ;bueno, la peor respuesta que se le podía ocurrir a alguien dar!, porque: Tú adoptas por tu propio egoísmo. Bueno, ¡ya!, por una parte, es verdad, ipero un puntito altruista también se puede tener! A ver, para mí la cosa era como que había muchísimo juicio, muchísima valoración, y había que responder con muchísimo cuidado, como ¡ojito, que te están evaluando! (EE19).

De esta forma, en sus relatos intentan tomar distancia, cuidando discursivamente no relacionarse con una motivación de corte solidario, dada su deslegitimización por parte del conocimiento experto como motivación funcional para adoptar, lo cual viene siendo debatido por algunos investigadores (Jociles y Charro, 2008; Jordana, 2009; Marre, 2009). Así mismo, encontramos narrativas que se distinguen y desmarcan explícitamente de una perspectiva caritativa de la adopción («No soy Santa Teresa de Calculta», «No soy una santa», «Eso no significa que vaya a hacer una obra de caridad»), que puede restarles legitimidad como madres y desdibujar el carácter recíproco y solidario que valoran de la relación construida con sus hijos. 
No quiero sentir que esto es una cosa de caridad hacia ellos. No lo he sentido nunca así, siento que (mi hija) me ha aportado más a mí de lo que yo le podría aportar a ella. ¿¿No sé si me entiendes? No hay caridad (ECH1).

¡A ver!, yo creo que ha influido [en haber adoptado] lo que he viajado, porque niños hay por todas partes. A ver, no me malinterpretes: yo no lo hago por caridad, por decir: ¡qué generosa soy! (EE13).

Contribuyendo a la complejidad de esta temática, otro relato muestra un discurso más salvacionista de la adopción, como el que se presenta a continuación.

Creo que mucha gente me admira por lo que he logrado con mis hijos, sobre todo con el primero, cómo lo saqué adelante luego de cómo lo recibí. (...) Es un niño muy complejo, tú lo ves hoy día y nada que ver con cuando recién llegó. (...) La adopción de mujeres solteras es un gesto de amor y generosidad mucho más grande, porque nosotras podríamos tener un hijo con cualquiera y hacernos cualquier tratamiento, pero decidimos adoptar niños en condiciones especiales (ECH2).

Estos verbatims permiten entrever la inconsistencia del discurso experto, que, por un lado, deslegitima la solidaridad — mal entendida como caridad como motivación para adoptar y, por el otro — desde una visión deficitaria de las adoptantes monoparentales y de los niños con estos perfiles_-, intenta «juntar a los menos deseados». De esta forma, se ejercen presiones sutiles o explícitas para que estas solicitantes adopten niños con problemas de salud graves, esperando una suerte de acto de renuncia y beneficencia que resuelva la problemática que entraña la adopción de niños con estos perfiles para el sistema de protección.

Me dicen: «iNo hay límites [en la discapacidad que tenga el niño]!». O sea, a ti te puede venir una niña que tenga una tara, y esa tara puede ser nada, que sea albina o que tenga un retardo o tal, o puede ser una niña que sea lisiada total. Y yo tenía clara una cosa, que santa no soy. Yo quería formar una familia, iy punto! Formar una familia no significa que vaya a hacer una obra de caridad, significa que quiero formar una familia y que, por lo tanto, yo a los riesgos de ser una niña adoptada, no tengo por qué añadirle, si puedo evitarlo, una falta de salud. No tenía por qué añadirle una serie de cosas simplemente porque yo soy soltera. Entonces, ahí me negué (EE3).

Retomando planteamientos anteriores (Jociles, 2013), entendemos que las negaciones, ambivalencias y/o deslindamientos de estas mujeres con respecto a la motivación de la solidaridad constituyen, en alguna medida, una estrategia desplegada para evitar el riesgo de no obtener la idoneidad si se desmarcan del discurso experto dominante sobre lo que es válido o esperable esgrimir como fundamento de su deseo y decisión de adoptar. No obstante, dicha deslegitimación contribuye también a construir un contexto de plausibilidad y acep- 
tación de actitudes discriminatorias hacia niños que presentan ciertos desafíos especiales atribuibles a su edad, su estado de salud o algún tipo de discapacidad.

A las inconsistencias y contradicciones del discurso experto ya mencionadas en torno a la temática, se suma otro elemento necesario de señalar: la escasez de apoyos institucionales pre y post-adoptivos. Más allá del seguimiento formal, no se aprecia una consistente disposición de recursos o apoyos formales durante la trayectoria adoptiva. Las entrevistadas relatan experiencias disímiles en cuanto a haber dispuesto de un acompañamiento profesional posterior o no haberlo tenido en absoluto, valiéndose casi exclusivamente de los recursos propios o de su entorno. Este es el caso de una adoptante chilena.

Me habría gustado tener una instancia un poco más formal, aunque sea libre, del tipo: una vez al mes nos vamos a juntar, vamos a compartir las experiencias. Encuentro que al final es como que te dicen: listo, y una se sorprende. O sea, arrégleselas, es su hijo, es suyo (ECH3).

Esto plantea la necesidad de construir una oferta accesible de apoyo durante la trayectoria adoptiva que aborde tanto los desafíos específicos de estas adopciones como también los comunes y compartidos con toda experiencia de parentalidad adoptiva, sirviendo de espacio de contención, soporte y aprendizaje colectivo, sin caer en segregaciones.

\section{Discusión y conclusiones}

El tipo de adopciones que abordamos son valoradas por las políticas, normativas y profesionales del campo de la adopción a partir de nociones a priori, en las que tanto los niños adoptables como las potenciales adoptantes son habitualmente vistos desde un enfoque de riesgo o deficitario. Los resultados cualitativos de los estudios analizados permiten reflexionar sobre diversos desafíos implicados en la transformación de las políticas y prácticas. En primer lugar, dado que ninguna característica del niño ni de la configuración familiar lleva per se al fracaso de la adopción, los desafiantes escenarios en el terreno de las adopciones nacionales e internacionales deberían continuar problematizando la propia noción de «adopciones especiales» y reformular las políticas y prácticas que afectan a unos niños que, de otra forma, corren el riesgo de ser considerados «inadoptables» (Avery, 2000). Además, parece necesario continuar problematizando la mirada carencial de las adopciones monoparentales, considerando que no se sostiene desde la evidencia científica. Resulta útil y necesario para los desafíos actuales abordar este tipo de adopciones fuera de estereotipos, prejuicios o estigmatizaciones, dejando atrás planteamientos basados a priori en "las deficiencias» asociadas a la estructura familiar y/o las características de los niños, que les atribuyen menor valor en el «mercado de la adopción» (Raleigh, 2012).

En segundo lugar, en ambos países observamos que la disposición a aceptar niños con estos perfiles es desde el primer contacto con las solicitantes valo- 
rada positivamente por los profesionales a cargo de los procesos evaluativos. Coincidiendo con Cousins (2003), prácticas tácitas o explícitas orientadas a la supuesta flexibilización de las expectativas de los solicitantes podrían resultar perjudiciales si se presiona a estas mujeres a adoptar a niños con perfiles muy disímiles a sus expectativas, deseos o motivaciones iniciales, más aun cuando esto ocurre sin fundamentos técnicos rigurosos que sirvan de base para ello o sin que se apoye en la evidencia científica disponible. En la conjugación de diversas variables, resultará de radical importancia la adecuación de expectativas, entendiendo que estas se reorganizan en un proceso formativo y reflexivo. La flexibilización de las expectativas en ningún caso debería valerse explícita o implícitamente de una valoración diferencial de la monoparentalidad, ni tampoco de la culpabilización o el sacrificio de aquellas solicitantes que no deseen o puedan hacerse cargo de un niño con determinadas necesidades o problemáticas adicionales.

En tercer lugar, consideramos importante establecer procesos de matching en función de lo que cada niño singularmente necesita y no de preconcepciones sobre cuál es la estructura familiar ideal para todo niño. La evaluación de idoneidad debería tratar de determinar no solo si quienes se proponen adoptar parecen ofrecer las condiciones necesarias para ello, sino también para qué tipo de niños, de qué edades y con qué otras características podrían ser idóneos, tomando para ello en consideración no solo la edad de los solicitantes de adopción o su estructura familiar para establecer el rango etario del niño adoptable, sino que lo que la evidencia señala idóneo para niños con determinadas características. Técnicamente hablando, una solicitante monoparental podría ser adecuada para hacerse cargo de un bebé, pero no de un niño mayor y, por el contrario, una pareja podría ser idónea para adoptar un niño mayor, pero no para otro más pequeño.

Los criterios «técnicos» subyacentes al «matching diferencial» que hemos analizado resultan contradictorios con el principio jurídico que pretende garantizar la elección de la mejor familia para un determinado niño, viéndose agravado por el énfasis meramente evaluativo del proceso pre-adoptivo y la casi inexistente oferta y disponibilidad de apoyos pre y post-adoptivos que superen la lógica de seguimiento-control. De esta forma, la operacionalización del «Interés Superior del Niño» para determinar cuál es la mejor familia para un niño en concreto debe realizarse fuera de las valoraciones deficitarias de la adopción monoparental, que actualmente orientan, a partir de una diferenciación ideológica de las estructuras familiares, la asignación de niños que son menos demandados en el «mercado adoptivo» a estas adoptantes. Ni la adopción monoparental ni la adopción de niños mayores o con determinadas problemáticas añadidas deben asociarse per se con mayores dificultades para el éxito de la misma, pero para ello también deben proporcionarse los apoyos oportunos y especializados para todos los implicados (Coakley y Berrick, 2008; O'Dell et al., 2015).

En cuarto lugar, consideramos contradictorio y problemático para el actual escenario adoptivo y los nuevos perfiles de niños y solicitantes, que la llamada 
«motivación por la solidaridad» continúe siendo considerada disfuncional por parte del discurso experto. La deslegitimación de plano de esta motivación y la equiparación del deseo de ayuda con «la caridad» o la beneficencia contribuyen directa o indirectamente a sostener actitudes y prácticas discriminatorias de ciertos niños en función de su edad, su estado de salud o su historia de origen. La aún hegemónica significación de la solidaridad aducida por los profesionales, e internalizada muchas veces por las adoptantes, reduce los posibles sentidos que puede llegar a tener este término, en tanto se lo disloque de la noción tradicional de caridad. En otras palabras, solidaridad no es sinónimo de caridad o de rescate. Con la primera se significa una relación en que ambas partes dan y reciben mutuamente - acercándose más a la idea de reciprocidad-, mientras que con la segunda se apunta a una relación basada en la idea de que alguien que tiene dona algo a alguien que no lo tiene. La solidaridad implica pensar que la adopción puede ser beneficiosa tanto para la persona adoptante como para el niño que será adoptado, que en toda relación familiar se produce el encuentro de diversas necesidades afectivo-amorosas, y que en ella la reciprocidad y solidaridad siempre están presentes.

En quinto lugar, identificamos que, al igual que los demás solicitantes de adopción, estas adoptantes suelen carecer de apoyos institucionales y profesionales más allá de lo informativo y evaluativo, por lo que se valen casi exclusivamente de sus recursos personales y privados. En los casos analizados, las adoptantes deben hacer frente a múltiples discriminaciones durante el proceso adoptivo y, al mismo tiempo, demostrar y sostener capacidades extraordinarias para responder incluso a la tarea de «reparar» aquellos daños derivados de la propia desarticulación de los sistemas de protección de la infancia que derivan en la transformación de los perfiles de los niños asignados. De este modo, se ubican en la situación paradójica de ser consideradas deficitarias con relación al modelo normativo de familia y, al mismo tiempo, ser sobreexigidas, haciéndolas cargo de asumir adopciones consideradas tanto por los investigadores como por los equipos técnicos como más complejas, sin contar con apoyos accesibles, oportunos y especializados.

Si bien es cierto que tanto en Chile como en España existen profesionales y recursos de apoyo post-adoptivo especializados, estos no son siempre públicos y/o gratuitos, con lo cual los solicitantes no pueden acceder a ellos si no es a costa de un significativo esfuerzo económico personal, acudiendo muchas veces a servicios y profesionales no necesariamente especializados en el campo de la adopción. Ni las políticas públicas chilenas ni las españolas contemplan actualmente los apoyos necesarios requeridos para incentivar la adopción de niños mayores o con determinadas «necesidades especiales», y menos aun por parte de solicitantes monoparentales. La ausencia de algunos servicios críticamente necesarios y los vacíos de ayudas públicas todavía representan una situación preocupante en tanto existen muchas familias adoptivas potencialmente desatendidas (Hartinger-Saunders y Trouteaud, 2015). Frente a ello, resulta urgente que los Estados se comprometan a brindar apoyos y recursos de diversa índole, en consideración de que — como se ha dicho- estas mujeres suelen 
trabajar a tiempo completo y no siempre cuentan con recursos suficientes para dejar de hacerlo al depender de un solo salario para sostener sus hogares, lo que podría adicionarles dificultades (económicas, de disponibilidad de tiempo o de recursos técnico-educativos) para poder atender ciertos requerimientos de sus hijos en razón, por ejemplo, de la edad en la que fueron adoptados o de otras necesidades específicas. En el caso español, se podría incluir, por ejemplo, la posibilidad de que la madre, una vez disfrutado el permiso de maternidad, pudiera acogerse también al de paternidad, que le supondría un aumento del tiempo de permiso en el caso de que el hijo adoptado mayor de 6 años tuviera «alguna dificultad especial para incorporarse con normalidad a la familia»; la existencia de una ley estatal de familias monoparentales que posibilitara que estas, por el solo hecho de ser sostenidas por un solo padre/madre, tuvieran acceso a los mismos beneficios que las familias numerosas, tales como la reducción del $45 \%$ de las cuotas de la Seguridad Social (SS), el incremento del límite de ingreso para tener derecho a la asignación económica de la SS por hijo a cargo menor de 18 años, preferencia para conseguir becas y bonificación del $50 \%$, o incluso la exención, en ciertos supuestos, de tasas y precios públicos en el ámbito de la educación, la reducción del $20 \%$ al $50 \%$ en las tarifas del transporte por carretera, ferrocarril y marítimo, etcétera (Ministerio de Sanidad, Servicios Sociales e Igualdad, 2016).

Por último, en lo que respecta a las limitaciones y proyecciones del presente trabajo, cabe señalar la necesidad y la importancia de complementar estos hallazgos con la perspectiva de otros actores claves, como los propios profesionales del sistema judicial y técnicos que intervienen en estos procesos ${ }^{7}$ y, muy especialmente, con aquellos niños susceptibles de ser adoptados, que se encuentran a la espera de una asignación, o bien que hayan sido adoptados por estas solicitantes, para conocer sus experiencias y perspectivas. Asimismo, sería interesante entrevistar en futuros trabajos a aquellas entrevistadas que han quedado fuera de las posibilidades de adoptar. Finalmente, es clave la difusión de estos resultados no solo en medios científico-académicos, sino también entre solicitantes o adoptantes, agrupaciones, instituciones y profesionales que trabajan en este campo, a quienes podría servir de material de reflexión sobre las ideologías y criterios técnicos construidos en torno al tema.

\section{Referencias bibliográficas}

AgAr, M. (2006). «An ethnography by any other name...». Forum: Qualitative Social Research, 7 (4), 36.

Amaral, N. R. do y Rossetti-Ferreira, M. C. (2009). «Becoming mother and father in late adoption: A case study». Child and Family Social Work, 14, 58-67. <https://doi.org/10.1111/j.1365-2206.2008.00581.x>

AOKI, S. (1977). «Single-parent adoption». Adoption \& Fostering, 89 (3), 35-39. <https://doi.org/10.1016/S0190-7409(00)00094-3>

7. En el caso de España, esto ha sido realizado por el equipo de investigación. 
BARTH, R. P. (1991). «Research on special needs adoption». Children and youth service review, 13, 317-321.

BEN-ARI, A. B. y WeINBERG-KURNIK, G. (2007). «The dialectics between the personal and the interpersonal in the experiences of adoptive single mothers by choice». Sex Rles, 56, 823-833.

BerÁstegui, A. (2003). Las adopciones truncadas y en riesgo en la Comunidad de Madrid. Madrid: Consejo Económico y Social de la Comunidad de Madrid.

BerÁstegui, A. (2012). «Adopciones especiales: ¿niños especiales para familias especiales?». Papeles del Psicólogo, 33 (3), 211-220.

AverY, R. J. (2000) «Perceptions and practice: Agency efforts for the hardest-to-place children». Children and Youth Services Review, 22 399-420.

Bock, J. D. (2000). «Doing the right thing? Single mothers by choice and the struggle for legitimacy». Gender and Society, 14 (1), 62-86.

Bourdieu, P. (1991). El sentido práctico. Madrid: Taurus.

Blackstone, E. A.; Bucka, A. J.; Hakima, S. y Spiegel, U. (2008) «Market segmentation in child adoption». International Review of Law and Economics, 28 (3), $220-225$. $<$ https://doi.org/10.1016/j.irle.2008.06.001>

BRIND, K. (2008). "An exploration of adopters' views regarding children's ages at the time of placement». Child and Family Social Work, 13, 319-328. <https://doi.org/10.1111/j.1365-2206.2008.00556.x>

BunT, S. (2013). «A framework for the analysis of the social processes in the adoption of disabled children». Journal of Social Work, 14 (5), 524-541. <https://doi.org/10.1177/1468017313478901>

Cadoret, A. (2003). Padres como los demás: homosexualidad y parentesco. Barcelona: Gedisa.

COAKLEY, J. F. y BERRICK, J. D. (2008). «Research review: In a rush to permanency. Preventing adoption disruption». Child and Family Social Work, 13, 101-112.

Cousins, J. (2003). «Are we missing the match? Rethinking adopter assessments and child profiling». Adoption and Fostering, 27, 4.

- (2009). «Placing disabled children with permanent new families». En: SCHOFIELD, G. y SimmONDS, J. (eds.). The child placement handbook, 345-363. Londres, UK: BAAF.

COWAN, A. B. (2004). «New strategies to promote the adoption of older children out of foster care». Children and Youth Services Review, 26 (11), 1007-1020. <http://doi.org/10.1016/j.childyouth.2004.08.002>

DíEZ, M.; Morgado, B. y GonZÁLEZ, M. M. (2016). «El apoyo social y la satisfacción vital, factores clave en el caso de las madres adoptivas solas». Apuntes de Psicología, 34 (2-3), 136-146.

DougherTy, J. (1978). «Single adoptive mothers». Social Work, 32, 311-314.

EBraHim, S. G. (2001). «Adocao tardia: altruismo, maturadidade e estabilidade emocional». Psicologia, Reflexao e Critica, 14 (1), 73-80.

Feigelman, W. y Silverman, A. R. (1977). «Single parent adoption». Social Casework, $58(7), 418-425$.

Forbes, H. y DZIEGIELEWSKI, S. (2003). «Issues facing adoptive mothers of children with special needs». Journal of Social Work, 3 (3), 301-320.

Galleguillos, M. F. (2015). «Adopción: interés superior del niño/a versus enfoque biologicista». En: Fernández, P.; Lara, A.; Oltra, S. y Riquelme, P. (comps.). Infancias, familias y Estado. Santiago de Chile: Ediciones UCSH. 
García, L. y Grau, J. (2016). «La cara oculta de la adopción. Las adopciones internacionales truncadas en Catalunya». Periferias, 21 (2), 29-57. $<$ https://doi.org/10.5565/rev/periferia.578>.

GONZÁlEZ, M. M.; JiMÉNEZ, I.; MORGADO, B. y DíEZ, M. (2007). Madres solteras por elección: análisis de la monoparentalidad emergente. Madrid: Instituto de la Mujer.

Groze, V. (1991). «Adoption and single parents. A review». Child Welfare League of America, LXX (3), 321-332.

Groze, V. y Rosenthal, J. (1991). «Single parents and their adopted children: A psychosocial analysis». Families in Society: Journal of Contemporary Human Services, $72(2), 67-77$.

Hanna, M. D. y Mcroy, R. G. (2011). «Innovative practice approaches to matching in adoption». Journal of Public Child Welfare, 5 (1), 45-66. <https://doi.org/10.1080/15548732.2011.542722>

Hartinger-Saunders, R. M. y Trouteaud, A. (2015). «Underserved adoptive families: Disparities in postadoption access to information, resources, and services». Journal of Family Strengths, 15 (1), 1-35.

Haugaard, J., Palmer, M. y Wojslawowicz, J. C. (1999). «Single-parent adoptions». Adoption Quarterly, 2 (4), 65-74. <https://doi.org/10.1300/J145v02n0405>

HÖGBACKA, R. (2008). "The quest for a child of one's own: Parents, markets and transnational adoption». Journal of Comparative Family Studies, 39 (3), 311-330.

JOCILES, M. I. (2013). «Resistiéndose ante el conocimiento experto: monoparentalidad adoptiva y tácticas para legitimar la solidaridad como motivación para adoptar». Cultura y educación, 25 (2), 213-228.

Jociles, M. I. y Charro, C. (2008). «Construcción de los roles paternos en los procesos de adopción internacional: El papel de las instituciones intermediarias». Politica y Sociedad, 45 (2), 105-130.

Jociles, M. I.; Rivas, A. M. y PovedA, D. (2012). «Las representaciones expertas sobre las solicitantes individuales en los procesos de adopción». Revista de Dialectología y Tradiciones Populares, LXVII (2), 535-558. $<$ https://doi.org/10.3989/rdtp.2012.21>

JORDANA, O. (2009). «Una aproximación antropológica a la maternidad voluntariamente sola en Barcelona». Revista de Antropología Experimental, 9 (7), 91-102.

Julian, M. (2013). "Age at adoption from institutional care as a window into the lasting effects of early experiences». Clinical Child and Family Psychology Review, 16 (2), 101-145. <https://doi.org/10.1007/s10567-013-0130-6>

KAGAN, R. M. y REID, W. J. (1986). «Critical factors in the adoption of emotionally disturbed youths». Child Welfare, 65 (1), 63-73.

LEUNG, P.; ERICH, S. y KANENBERG, H. (2005). «A comparison of family functioning in gay/lesbian, heterosexual and special needs adoptions». Children and Youth Services Review, 27, 1031-1044. $<$ https://doi.org/10.1016/j.childyouth.2004.12.030>

Marre, D. (2009) «Los silencios de la adopción en España». Revista de Antropología Social, 18, 97-126.

Mcroy, R. G. (1999). Special needs adoptions: Practice Issues. Nueva York: Garland Publishing, Inc.

Medina, R. (2013). «Imbricación del discurso jurídico y el discurso experto en torno a la idoneidad para la adopción en familias monoparentales». En: JociLEs, M. I. 
y Medina, R. La monoparentalidad por elección. El proceso de construcción de un modelo de familia. Valencia: Tirant Lo Blanch.

Memmi, D. (2014). La revanche de la chair. Essai sur les nouveaux supports de l'identité. París: Seuil.

MSSSi (Ministerio de Sanidad, Seguridad Social e Igualdad (2016). Guía de Ayudas Sociales. Madrid: MSSSI.

MSSSI (Ministerio de SANidad, Seguridad Social E Igualdad) (2017). Estadísticas de Adopción Internacional 2011-2015. Madrid, España. Recuperado de <http://www.msssi.gob.es/ssi/familiasInfancia/Infancia/adopciones/2015ESTA DISTICA20112015.pdf>.

- (2017b). Boletín de datos estadisticos de medidas de protección a la infancia, $n^{\circ} 18$. Datos de 2015. Madrid, España. Recuperado de <http://www.msssi.gob.es/ssi/ familiasInfancia/Infancia/pdf/Boletinproteccion 18provisionalcorrecto.pdf>.

O’Dell, K. E.; MCCALl, R. B. y GroarK, C. J. (2015). «Supporting families throughout the international special needs adoption process». Children and Youth Services Review, 59, 161-170. $<$ https://doi.org/10.1016/j.childyouth.2015.11.008>.

OTUKA, L.; SCORSOLINI-COMIN, F. y SANTOS, M. A. dos. (2013). «Adoção tardia por casal divorciado e com filhos biológicos: novos contextos para a parentalidade». Estudos de Psicologia, 30 (1), 89-99.

Palacios, J. (2007). «Después de la adopción: necesidades y niveles de apoyo». Anuario de Psicología, 38 (2), 181-198.

Paniagua, C.; Jiménez-Morago, J. M., y Palacios, J. (2016). «Adopciones rotas en Andalucía: caracterización y propuestas para la intervención». Apuntes de Psicología, 34 (2-3), 301-309.

PAKIZEGI, B. (2007). «Single-parent adoptions and clinical implications». En: RAFAEL JaVier, A.; BADEN, A.; BiAFOra, F. y CAMACHO-GINGERICH, A. (eds.). Handbook of adoption. Implications for researchers, practitioners, and families (190-216). CA: Sage.

- (2012). «Adolescent adoptees of single mothers by choice». International Journal of Humanities and Social Science, 2 (1), 173-188.

PENA, M. (2016). «Relatos de adopciones monoparentales: tensiones y potencialidades». Revista Interdisciplinaria de Estudios de Género de El Colegio de México, 2 (4), 103-125.

PovedA, D.; Jociles, M. I.; Rivas, A. M. y LoRes, F. (2013). «Discurso experto sobre la idoneidad e instrumentalización del interés superior del menor en la valoración de las familias monoparentales». En: Jociles, M. I. y MedinA, R. (ed.). La monoparentalidad por elección. El proceso de construcción de un modelo de familia (267-292). Valencia: Tirant Lo Blanch.

Raleigh, E. (2012). "Are same-sex and single adoptive parents more likely to adopt transracially? A national analysis of race, family structure, and the adoption marketplace». Sociological Perspectives, 55 (3), 449-471.

RiESSMAN, C. K. (2008). Narrative methods for the human sciences. Londres: Sage.

RodrígueZ, M. J. y JAREÑO, D. (2015). «Estigma social y adopción internacional en España. ¿Es la familia adoptiva un modelo familiar menos «auténtico» que los basados en lazos biológicos?». Papers, 100 (2), 211-236. $<$ https://doi.org/10.5565/rev/papers.2070>

Rosenthal, J. A.; SCHMidT, D. y Conner, J. (1988). «Predictors of special needs adoption disruption: An exploratory study». Children and Youth Services Review, $10,101-117$. 
Rosser, A. y Moya, C. (2001). «Familias monoparentales e idoneidad para la adopción». Intervención Psicosocial, 10 (2), 209-220.

Salvo Agoglia, I. (2018). «Narrativas de mujeres que adoptan monoparentalmente en Chile: dispositivos de intervención y prácticas de resistencia». Revista de Estudios Sociales, 63, 29-41. <https://doi.org/10.7440/res63.2018.03>

SELman, P. (2012). "The global decline of intercountry adoption: What lies ahead?». Social Policy and Society, 11 (3), 381-397. $<$ https://doi.org/10.1017/s1474746412000085>

- (2016). "The rise and fall of intercountry adoption in the 21 st century». International Social Work, 52 (5), 575-594. $<$ https://doi.org/10.1177/0020872809337681>

SENAME (2018). Anuario estadístico 2017. Servicio Nacional de Menores: Chile. Recuperado de <http://www.sename.cl/web/anuarios-estadisticos-sename/>.

Shireman, J. F. (1994). «Adoptions by single parents». Marriage \& Family Review, $20(3-4), 367-388$. <https://doi.org/10.1300/J002v20n0304>

Shireman, J. F. y Johnson, R. R. (1985). «Single parent adoptions: A longitudinal study». Children \& Youth Services Review, 7, 321-334.

Silva, C. y Benetti, S. (2015). "Older child adoption: A study of the affiliation process». Estudos de Psicologia (Campinas), 32 (1), 121-127. <https://doi.org/10.1590/0103-166X2015000100011>

STRAUSS, A. y CORBIN, J. (1998). Basics of qualitative research techniques and procedures for developing grounded theory. Londres: Sage Publications.

TAN, T. X. y BAGGERLY, J. (2009). «Behavioral adjustment of adopted chinese girls in single-mother, lesbian-couple, and heterosexual-couple households». Adoption Quarterly, 12 (3-4), 171-186. <https://doi.org/10.1080/10926750903313336>.

Van Laningham, J. L.; Scheuble, L. K. y Johnson, D. R. (2012) «Social factors predicting women's consideration of adoption». Michigan Family Review, 16 (1), $1-21$.

VAsilachis de Gialdino, I. (2009). Estrategias de investigación cualitativa. Buenos Aires: Gedisa. 